\title{
Improving the Performance of MO-RV-GOMEA on Problems with Many Objectives using Tchebycheff Scalarizations
}

\author{
Ngoc Hoang Luong \\ Centrum Wiskunde \& Informatica \\ Amsterdam, The Netherlands \\ Hoang.Luong@cwi.nl
}

\author{
Tanja Alderliesten \\ Academic Medical Center \\ Amsterdam, The Netherlands \\ T.Alderliesten@amc.uva.nl
}

\author{
Peter A. N. Bosman \\ Centrum Wiskunde \& Informatica \\ Amsterdam, The Netherlands \\ Peter.Bosman@cwi.nl
}

\begin{abstract}
The Multi-Objective Real-Valued Gene-pool Optimal Mixing Evolutionary Algorithm (MO-RV-GOMEA) has been shown to exhibit excellent performance in solving various bi-objective benchmark and real-world problems. We assess the competence of MO-RVGOMEA in tackling many-objective problems, which are normally defined as problems with at least four conflicting objectives. Most Pareto dominance-based Multi-Objective Evolutionary Algorithms (MOEAs) typically diminish in performance if the number of objectives is more than three because selection pressure toward the Pareto-optimal front is lost. This is potentially less of an issue for MO-RV-GOMEA because its variation operator creates each offspring solution by iteratively altering a currently existing solution in a few decision variables each time, and changes are only accepted if they result in a Pareto improvement. For most MOEAs, integrating scalarization methods is potentially beneficial in the many-objective context. Here, we investigate the possibility of improving the performance of MO-RV-GOMEA by further guiding improvement checks during solution variation in MO-RV-GOMEA with carefully constructed Tchebycheff scalarizations. Results obtained from experiments performed on a selection of well-known problems from the DTLZ and WFG test suites show that MO-RVGOMEA is by design already well-suited for many-objective problems. Moreover, by enhancing it with Tchebycheff scalarizations, it outperforms MOEA/D-2TCHMFI, a state-of-the-art decompositionbased MOEA.
\end{abstract}

\section{CCS CONCEPTS}

- Mathematics of computing $\rightarrow$ Evolutionary algorithms;

\section{KEYWORDS}

Multi-objective optimization, Tchebyche scalarization

\section{ACM Reference Format:}

Ngoc Hoang Luong, Tanja Alderliesten, and Peter A. N. Bosman. 2018. Improving the Performance of MO-RV-GOMEA on Problems with Many Objectives using Tchebycheff Scalarizations. In GECCO '18: Genetic and Evolutionary Computation Conference, July 15-19, 2018, Kyoto, Japan. ACM, New York, NY, USA, 8 pages. https://doi.org/10.1145/3205455.3205498

Permission to make digital or hard copies of all or part of this work for personal or classroom use is granted without fee provided that copies are not made or distributed for profit or commercial advantage and that copies bear this notice and the full citation on the first page. Copyrights for components of this work owned by others than ACM must be honored. Abstracting with credit is permitted. To copy otherwise, or republish, to post on servers or to redistribute to lists, requires prior specific permission and/or a fee. Request permissions from permissions@acm.org.

GECCO '18, July 15-19, 2018, Kyoto, Japan

(C) 2018 Association for Computing Machinery.

ACM ISBN 978-1-4503-5618-3/18/07 . \$ \$15.00

https://doi.org/10.1145/3205455.3205498

\section{INTRODUCTION}

The Multi-Objective Real-Valued Gene-pool Optimal Mixing Evolutionary Algorithm (MO-RV-GOMEA), a recently-introduced member of the GOMEA family, is a state-of-the-art Multi-Objective Evolutionary Algorithm (MOEA) for the continuous domain that exhibits both superior scalability on benchmark problems [6] and excellent performance on real-world applications [16]. MO-RVGOMEA is characterized by three main features. First, explicit linkage models that align with dependencies among decision variables of the problem instance under consideration are either constructed during optimization by performing linkage learning on the working population (in black-box optimization), or are defined $a$ priori by using available problem-specific knowledge (in gray/white-box optimization). Second, a variation operator, named Gene-pool Optimal Mixing (GOM), is used to exploit the learned linkage information during solution variation in an effective manner such that offspring solutions are guaranteed to have equal or better fitness values than parent solutions. Third, a niching concept is realized in MO-RVGOMEA by a cluster-based operation, i.e., in every generation, solutions in the population are partitioned into equal-sized clusters; linkage learning and solution variation can then be performed per cluster, thereby increasing the probability that all different regions of the Pareto-optimal front are evenly approached.

A key feature of GOMEAs is that the GOM variation operator of GOMEAs transforms an existing solution into an offspring solution through a series of so-called mixing events. Each mixing event is a partial solution alteration that involves only decision variables that are dependent on each other to some degree (as indicated by the learned, or a priori defined, linkage models). Each partially-altered solution is then evaluated for improvement and the new decision variable values are only accepted if the fitness value of the solution does not deteriorate. This native genetic-local-search-like variation operator makes partial evaluations straightforward to be implemented in GOMEAs if the problem permits efficient computation of the effect of changing values of a few decision variables. For many other Evolutionary Algorithms (EAs), such partial evaluations are non-trivial or impractical to be implemented because a whole offspring solution is created each time without intermediate improvement checks as in GOM. Partial evaluations substantially increase the performance of MO-RV-GOMEA, especially in realworld applications where each full solution evaluation typically incurs a considerable amount of computation cost (e.g., see [16]).

However, while succeeding in solving complicated problems with thousands of decision variables, MO-RV-GOMEA has mainly been tested on bi-objective optimization problems $[6,16]$. The performance of MO-RV-GOMEA on problems that have more than two 
objectives is yet to be investigated. Especially the class of manyobjective problems, which is often defined as problems that have at least four objectives, poses substantial challenges for many MOEAs. The primary difficulty is that as the number of objectives increases, using the Pareto dominance relation in selection based on an entire population does not effectively maintain (selection) pressure toward the Pareto-optimal front since most solutions in the population of an MOEA are non-dominated $[1,12]$.

In recent years, the class of decomposition-based MOEAs has emerged as a more effective methodology for tackling multi- and especially many-objective problems. A decomposition-based MOEA typically decomposes a multi-objective problem into a set of singleobjective optimization subproblems, in which each subproblem is a scalarization of the original problem, i.e., aggregating all objectives on the basis of a unique weight vector. A diverse set of weight vectors is normally used to create a well-spread set of scalarization functions, aiming to evenly approximate the Pareto-optimal front. Selection pressure is maintained by using scalar improvements associated with the scalarization functions (which are similar to single-objective optimization) rather than depending on Pareto dominance improvements. The well-known MOEA based on Decomposition (MOEA/D) [19] presents three options for performing aggregation: the weighted-sum approach, the weighted Tchebycheff method, and the Penalty-based Boundary Intersection (PBI) method. The latter two are more commonly used since they are able to obtain solutions on non-convex regions, if such exist, of Pareto-optimal fronts. The Tchebycheff method is more convenient to use since it does not require the user to define the penalty factor $\theta$ as in the PBI method, which can have significant influence on the performance of the search [12]. Many MOEA/D variants have been proposed to provide improvements on several aspects of the original MOEA/D [22]. The recently-introduced Nondominated Sorting Genetic Algorithm III (NSGA-III) [9] also employs weight vectors in the form of reference points to improve the performance of the well-known NSGA-II when solving many-objective problems. Therefore, in this paper, besides benchmarking MO-RV-GOMEA in the many-objective realm, we also investigate the possibility of employing the Tchebycheff method to enhance the performance of the original MO-RV-GOMEA.

The remainder of the paper is organized as follows. In Section 2, we outline MO-RV-GOMEA. In Section 3, we propose our approach to integrate the Tchebycheff aggregation function into MO-RVGOMEA. Optimization problems employed for benchmarking and our experiment settings are described in Section 4. Results are discussed in Section 5. Finally, conclusions are drawn in Section 6.

\section{MO-RV-GOMEA OUTLINE}

MO-RV-GOMEA maintains a population $P$ of $N$ candidate solutions (i.e., population members), which can be randomly initialized, as well as an elitist archive to keep track of non-dominated solutions obtained during the search because elitism is beneficial to convergence [13]. Until the termination criteria are satisfied, the following procedure is performed in every generation. First, non-dominated ranks of all solutions are determined and truncation selection is performed to select a set $S$ that consists of $\lfloor\tau N\rfloor$ best ranked solutions from $P$ ( $\tau=0.35$ in [6]). Second, the selected solutions in $S$ are then partitioned into $k$ clusters, and another $m$ clusters are taken from the population ( $m$ is the number of objectives). Third, for each cluster, linkage learning is performed to construct a linkage model $\mathcal{F}$ consisting of a set of linkage sets on the basis of the solutions in that cluster. A Gaussian distribution is estimated for each linkage set in the linkage model. Next, the set of estimated Gaussian distributions associated with each cluster is used to iteratively construct offspring solutions in a step-wise manner.

\subsection{Clustering}

In every generation, $q=k+m$ clusters of candidate solutions are formed. First, $k$ leader solutions that are far apart from each other are selected from $S$ by a nearest-neighbor heuristic as follows.

Step 1: The solution that has the maximum value for a randomly chosen objective is assigned as the first leader. The Euclidean distances in objective space from all the remaining solutions to the first leader are computed and stored as their nearest-neighbor distances.

Step 2: The solution with the largest nearest-neighbor distance is assigned as the next leader.

Step 3: The Euclidean distance from each remaining solution to the newly assigned leader is computed and the nearest-neighbor distance is updated if the new distance is smaller than the currently stored value. Go back to Step 2 until $k$ leaders are obtained.

These $k$ leaders are then assigned as the centroids of the $k$ clusters. From its centroid, each cluster is formed by expanding to cover its $c$ nearest solutions. These $k$ clusters, named middle clusters, will then be used for multi-objective dominance-based optimization. For the remaining $m$ clusters, each of them is associated with a unique objective $i$ and is formed by selecting from the population $P$ the best $c$ solutions according to the corresponding objective $i$. These $m$ clusters, named extreme clusters, will then be used for single-objective scalar optimization along the associated objectives. All these $q$ clusters are called selection clusters (where each cluster $C$ has $c$ members) as they are formed by selection procedures, and it was recommended to have $c=\frac{2}{q}|S|[6]$.

\subsection{Linkage Learning}

Let $L=\{1,2, \ldots, l\}$ be the set that contains the indices of all $l$ decision variables. For each cluster, a linkage model $\mathcal{F}$ that describes the dependencies among $l$ decision variables is constructed. The Family-Of-Subset (FOS) concept is used as the general linkage model. Each FOS is a set of linkage sets $\mathcal{F}=\left\{\mathbf{F}^{1}, \mathbf{F}^{2}, \ldots, \mathbf{F}^{|\mathcal{F}|}\right\}$, in which linkage set $\mathbf{F}^{i} \subseteq L$ contains the indices of the decision variables that are dependent on each other to some degree. The two most common FOS structures in GOMEA literature are: 1) the univariate model, in which all variables are considered totally independent; and 2) the linkage tree model, a hierarchical ordering of linkage sets in which decision variables can be considered independent according to some linkage sets and can be considered dependent according to some other linkage sets. The linkage tree model can thus express hierarchical dependencies between problem variables. The goal is to configure the linkage model $\mathcal{F}$ such that it aligns with the variable-dependence problem structure of the problem instance under concern. If problem-specific knowledge is available, $\mathcal{F}$ can be constructed a priori. Otherwise, $\mathcal{F}$ can be learned by linkage learning algorithms (see [6] for details). 
For each linkage set $\mathbf{F}^{i}$ in the (learned) linkage model $\mathcal{F}$ of each selection cluster $C$, the parameters of a Gaussian distribution, that involves the variables encoded in $\mathbf{F}^{i}$, are estimated over the cluster solutions. Thus, a univariate Gaussian distribution (i.e., mean and variance) is estimated for a univariate linkage set, and a multivariate Gaussian distribution (i.e., mean vector and covariance matrix) is estimated for a multivariate linkage set. In order to alleviate the potential premature diminishing of variance due to selection, the variance parameters of these Gaussian distributions are proportionally scaled with the distance of improvements found from the Gaussian means. These Gaussian distributions are then used to sample new values during solution variation (more details in [6]).

\subsection{Gene-pool Optimal Mixing (GOM)}

Each solution $\boldsymbol{x}$ in the population is associated with the nearest selection cluster (based on the distance between $x$ and the cluster means in the objective space). This association effectively partitions the population into $q$ clusters, where each population cluster $C^{P}$ corresponds to a selection cluster $C$. A variation operator named GOM is employed to transform each solution $x \in C^{P}$ into an offspring $\boldsymbol{o}$ using the linkage model $\mathcal{F}$ of cluster $C$.

Instead of generating a whole offspring in one step as in many MOEAs, GOM constructs $\boldsymbol{o}$ in a step-wise manner as follows. All linkage sets in $\mathcal{F}$ of each cluster $C$ are iteratively considered in a random order. For each linkage set $F^{i}$, its associated Gaussian distribution is used to sample new values, altering all solutions of $C^{P}$ at the variables indicated by $\boldsymbol{F}^{i}$. These variables of a number of solutions, specifically $\left\lfloor\frac{1}{2} \tau\left|C^{P}\right|\right\rfloor$, are then further moved in the direction that the cluster mean is shifted in from the previous generation to the current generation [6]. The partially-altered solutions are evaluated for improvement. If $C$ is a middle cluster, each improvement check is based on Pareto dominance. That is, the change is accepted when the altered solution dominates the previous state or it can be accepted into the elitist archive. If $C$ is an extreme cluster, the change is accepted when the altered solution improves the previous state with respect to the objective associated with that extreme cluster. If no improvement is yielded, the change is revoked and the solution returns to its previous state. An offspring solution $\boldsymbol{o}$ is fully constructed after all linkage sets in $\mathcal{F}$ are considered.

The elitist archive $A$ of MO-RV-GOMEA is bounded by a userdefined target size. An adaptive mechanism maintains the archive around the target size by discretizing the objective space into equalsized hypercubes and only retaining one archive member per hypercube [17]. A solution $\boldsymbol{p}$ is accepted into the archive if it is not dominated by the archive ( $A \npreceq \boldsymbol{p}$ ), i.e., $\boldsymbol{p}$ is not dominated by any archive member and the hypercube that $\boldsymbol{p}$ resides in is unoccupied or $\boldsymbol{p}$ dominates the current resident member of that hypercube. After every generation, the hypercube sizes are adapted based on the range of the so-far-obtained Pareto front and the target size.

\section{MO-RV-GOMEA ADAPTATIONS}

\subsection{Tchebycheff Scalarization Method}

The weighted Tchebycheff distance (TCH) between a point $z$ and a given point $z^{*}$ in an $m$-dimensional space is defined as follows:

$$
\operatorname{TCH}\left(z, z^{*}, \boldsymbol{w}\right)=\max _{1 \leq i \leq m}\left\{w_{i}\left|z_{i}-z_{i}^{*}\right|\right\}
$$

where $\boldsymbol{w}=\left(w_{1}, \ldots, w_{m}\right)$ is a weight vector with $w_{i} \geq 0$ and $\sum_{i=1}^{m} w_{i}=1$.

A multi-objective problem with $l$ decision variables and $m$ objective functions can be described as follows:

$$
\min _{\boldsymbol{x} \in \Omega_{x}} f(\boldsymbol{x})=\left(f_{1}(\boldsymbol{x}), \ldots, f_{m}(\boldsymbol{x})\right)
$$

where $\boldsymbol{x}$ is a decision variable vector in the decision space (that we assume to be real-valued in this paper), $\Omega_{x} \subseteq \mathbb{R}^{l}, f=\left(f_{1}, \ldots, f_{m}\right)$ : $\Omega_{x} \rightarrow \Omega_{f}$ is the $m$-dimensional objective function vector, and $f(x)$ is thus the objective value vector of $x$ in the objective space $\Omega_{f} \subseteq \mathbb{R}^{m}$ of the multi-objective problem.

Using the weighted Tchebycheff distance with a utopian point as the point $z^{*}\left(z_{i}^{*} \leq \min \left\{f_{i}(\boldsymbol{x}) \mid \boldsymbol{x} \in \Omega_{x}\right\}\right)$, a multi-objective problem can be decomposed into a set of scalar optimization subproblems. Each subproblem has a unique weight vector $\boldsymbol{w}$ and is defined as:

$$
\min _{x \in \Omega_{x}}\left\{T C H\left(f(\boldsymbol{x}), z^{*}, \boldsymbol{w}\right)\right\}=\min _{x \in \Omega_{x}}\left\{\max _{1 \leq i \leq m}\left\{w_{i}\left|f_{i}(\boldsymbol{x})-z_{i}^{*}\right|\right\}\right\}
$$

It is known that with Tchebycheff scalarizations it is possible to identify solutions on potential non-convex regions of the Paretooptimal front [19] while this is not possible using linearly weighted sum scalarizations [7]. Therefore, Tchebycheff scalarizations are often employed in decomposition-based MOEAs.

\subsection{Weight Vector Generation}

Weight vectors are often generated such that they are spread as well as possible. Perfectly scattering $N$ points in a $d$-dimensional space $(d>1)$, however, is NP-hard [5]. Here, we employ a simple, yet effective, method to approximately generate $N$ well-spread weight vectors as follows. During initialization, we uniformly randomly generate $M$ weight vectors such that $M>>N$, where each weight vector $\boldsymbol{w}$ satisfies $w_{i} \geq 0$ and $\sum_{i=1}^{m} w_{i}=1$. We then apply the same heuristic that is used to select $k$ leaders in the $k$-leader-means clustering [4] (see Section 2) to select $N$ vectors that are spread as well as possible. An advantage of this approach is that any number of weight vectors $N$ can be generated rather than being restricted to some required numbers as in other methods (e.g., $[8,14])$.

\subsection{Weight Vector Association}

We employ the following procedure to associate each weight vector $\boldsymbol{w}$ with a candidate solution $\boldsymbol{x}$ in the current population $P$.

Step 1: Compute the ideal objective vector $z^{P}=\left(z_{1}^{P}, \ldots, z_{m}^{P}\right)$ of the current population $P$ on the basis of the solutions in $P$ :

$$
z_{i}^{P}=\min \left\{f_{i}(x) \mid x \in P\right\}
$$

Step 2: For each weight vector $\boldsymbol{w}$, compute the weighted Tchebycheff distance with $\boldsymbol{w}$ between each solution in the current population $P$ and the ideal objective vector $z^{P}$.

Step 3: Associate each weight vector $w$ with the nearest solution that has not been associated yet with any weight vector, based on the Tchebycheff distances that are computed with $w$ in Step 2 .

Figure 1a shows an example that illustrates the weight vector association procedure. In every generation, our procedure above explicitly assigns anew to each solution a unique improvement region (e.g., see Figure 1b). If a solution is not improved by GOM, and still remains in the next generation, it might be assigned a different improvement region. For many algorithms in the MOEA/D family, 


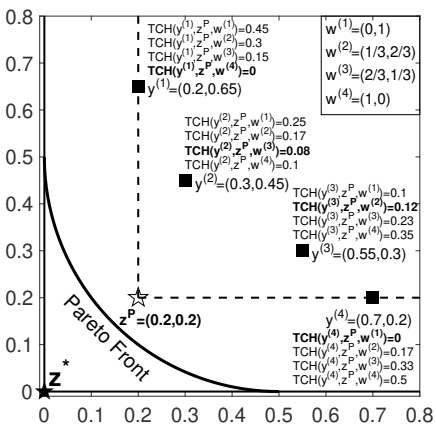

a) Tchebycheff scalarizations

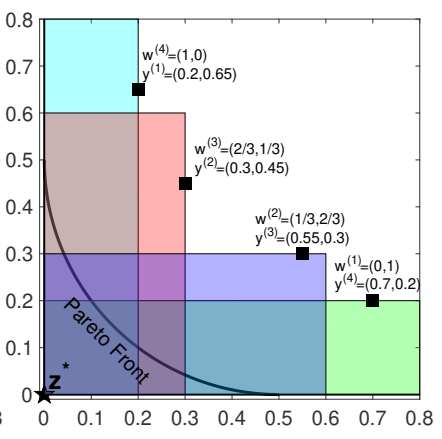

b) Improvement regions

Figure 1: x-y axes: objectives $\left(f_{1}, f_{2}\right)$. a) Weighted Tchebycheff distances between 4 points $y^{(i)}$ 's and the ideal objective point $z^{P}$ with 4 weight vectors $w^{(i)}$ 's, $i=1, \ldots, 4$. Bold-faced distances indicate the association between a vector $w$ and a point $y$. b) Improvement region of each solution defined by the associated weight vector $w^{(i)}$ and the utopian point $z^{*}$.

each solution is regarded as the best solution obtained so far for each subproblem. Therefore, if a solution is not changed in this generation (i.e., no offspring succeeds to replace the parent), its improvement region is still the same in the next generation. We note that there exist other weight vector association procedures in several weight vector-based MOEAs (e.g., NSGA-III [9], I-DBEA [1]). However, the association procedures in these MOEAs are mainly used for niching or diversity preservation purposes. Our association procedure here determines the improvement region for each solution and thus puts more emphasis on the exploitation purpose.

\subsection{Improvement Check in GOM}

Similar to the original MO-RV-GOMEA, each existing solution $\boldsymbol{x}$ is improved by the GOM variation operator with the learned linkage model of the cluster to which $\boldsymbol{x}$ belongs. At every mixing event, the partially-altered solution is evaluated and checked for improvement. In the original MO-RV-GOMEA, the improvement check is based on the Pareto dominance improvement for the solutions in the middle clusters, and is based on the scalar improvement for the solutions in the extreme-region clusters. The latter is thus already a form of Tchebycheff scalarization where the weight vector $w$ has weight $w_{i}=1$ for objective $i$ and weight 0 for the other objectives.

We propose two options to adapt the improvement check at each mixing event in GOM with Tchebycheff scalarizations as follows:

- Option 1: The change is accepted if the partially-altered solution $\boldsymbol{p}$ dominates the previous state $\boldsymbol{b}$, or $\boldsymbol{p}$ can be accepted into the elitist archive, or the objective value vector of $p$ has a smaller Tchebycheff distance (computed with the associated weight vector $w$ ) to the utopian point $z^{*}$ (compared to that of the previous state), i.e., $\boldsymbol{p} \leq \boldsymbol{b} \vee A \npreceq \boldsymbol{p} \vee T C H\left(f(\boldsymbol{p}), z^{*}, \boldsymbol{w}\right)<$ $T C H\left(f(b), z^{*}, w\right)$.

- Option 2: The change is accepted if $\boldsymbol{p}$ dominates $\boldsymbol{b}$ or the new objective value vector has a smaller Tchebycheff distance to the utopian point, i.e., $\boldsymbol{p} \leq \boldsymbol{b} \vee T C H\left(f(\boldsymbol{p}), z^{*}, \boldsymbol{w}\right)<$ $\operatorname{TCH}\left(f(b), z^{*}, w\right)$.
Note that the utopian point $z^{*}$ employed for the improvement check is different from the population's ideal objective vector $z^{P}$ employed for the weight vector association in Section 3.3. We here assume that the utopian point $z^{*}$ is known for the sake of convenience. In the case that the utopian point cannot be determined $a$ priori, $z^{*}$ can be approximated during optimization by updating $z_{i}^{*}$ to the best so-far-obtained value of objective $i$, if necessary, before computing the Tchebycheff distances in each improvement check.

\section{BENCHMARK AND EXPERIMENT SETTING}

\subsection{Benchmark Problems}

We perform experiments on two well-known sets of benchmark problems: DTLZ1-DTLZ4 [10] and WFG1-WFG4 [11]. For all problems, the number of decision variables is scaled as $l=40,80,160$, and 320. For DTLZ problems, we employ problem instances of $m=3,5,10$, and 15 objectives. For WFG problems, we employ problem instances of $m=2,3,5$, and 10 objectives, where the number of position parameters and the number of distance parameters are determined as in [2]. The reference Pareto-optimal fronts for both DTLZ and WFG problem instances are created by using the PlatEMO platform [21] such that a reference front with approximately $m \times 1000$ well-distributed points is generated for a problem instance with $m$ objectives.

\subsection{Experiment Setting}

We benchmark the original MO-RV-GOMEA and its two new adaptations with Tchebycheff scalarizations. For the purpose of comparisons, we also experiment with the recently-introduced MOEA/D2TCHMFI [18], an MOEA/D variant that employs the Tchebycheff scalarization with $l_{2}$-norm constraint, which is defined as

$$
\left.\operatorname{TCH}\left(\boldsymbol{f}(\boldsymbol{x}), z^{*}, \boldsymbol{w}\right)\right\}=\max _{1 \leq i \leq m}\left\{\frac{\left|f_{i}(\boldsymbol{x})-z_{i}^{*}\right|}{w_{i}}\right\}
$$

where $\boldsymbol{w}$ is a weight vector with $w_{i} \geq 0$ and $\|\boldsymbol{w}\|=1$.

The population size is a crucial control parameter for EAs, and it is also notoriously difficult to determine an appropriate population size for an EA solving a specific problem instance. While EA benchmark literature often uses the same population size for all algorithms, we argue that such a simple setting might not be proper because different EAs have different minimally-required population sizes, and the differences can be substantial (e.g., see [20]). Therefore, in this work, we employ the so-called Interleaved Multi-start Scheme (IMS) [6] (which originates from the populationsizing-free scheme of the Parameter-less Genetic Algorithm [15]) to adapt the population size during optimization for all MOEAs. The IMS operates multiple instances of an MOEA with different population sizes in an interleaved fashion. The initial population $P_{0}$ is started with a small population size $N_{0} . N_{0}$ can be any reasonably small population size. Here, we simply set $N_{0}=10 \times(m+1)$. A larger population $P_{i}$ with size $N_{i}=2 \times N_{i-1}$ is started later, and each generation of $P_{i}$ is then operated for every $g$ generations of the previous population $P_{i-1}$. A smaller population is deemed inefficient, and is thus terminated, if it contributes less than $10 \%$ of the combined front of non-dominated solutions obtained from all the working populations. All the populations are kept growing and running until the computation budget is spent. In this paper, 
Table 1: Medians and interdecile ranges (in brackets) of the IGD metric of the approximation fronts obtained by MOEAs on solving DTLZ problems. MOEA/D, GOMEA, Option 1, and Option 2 should be read as MOEA/D-2TCHMFI, the original MO-RVGOMEA, MO-RV-GOMEA with adaptation Option 1, and MO-RV-GOMEA with adaptation Option 2, respectively.

\begin{tabular}{|c|c|c|c|c|c|c|c|c|c|c|c|c|c|c|c|c|}
\hline & & $l=$ & $=40$ & & & & 80 & & & $l=1$ & & & & $l=3$ & & \\
\hline $\mathrm{m}$ & MOEA/D & GOMEA & Option 1 & Option 2 & MOEA/D & GOMEA & Option 1 & Option 2 & MOEA/D & GOMEA & Option 1 & Option 2 & MOEA/D & GOMEA & Option 1 & Option 2 \\
\hline & $0.043(0.729)$ & $0.289(1.177)$ & \begin{tabular}{|l|}
$0.008(0.003)$ \\
\end{tabular} & $0.008(0.002)$ & $0.104(10.489)$ & $1.155(2.338)$ & \begin{tabular}{|l|}
$0.008(0.004)$ \\
\end{tabular} & $0.007(0.002)$ & $0.560(5.451)$ & $1.877(4.069)$ & \begin{tabular}{|l|}
$0.008(0.009)$ \\
\end{tabular} & $0.008(0.003)$ & $13.606(61.756)$ & $4.544(4.475)$ & \begin{tabular}{|l|}
$0.008(0.571)$ \\
\end{tabular} & $0.008(0.002)$ \\
\hline & $0.785(8.820)$ & $0.034(0.002)$ & \begin{tabular}{|l|}
$0.035(0.002)$ \\
\end{tabular} & $0.035(0.002)$ & $3.593(23.129)$ & $0.034(0.003)$ & \begin{tabular}{|l|}
$0.034(0.003)$ \\
\end{tabular} & $0.035(0.002)$ & $18.648(50.245)$ & $\mathbf{0 . 0 3 3}(0.002)$ & \begin{tabular}{|l|}
$0.034(0.004)$ \\
\end{tabular} & $0.035(0.0 c$ & $58.282(136.725)$ & $0.033(0.003)$ & $0.035(0.005)$ & $0.035(0.002)$ \\
\hline & $2.903(18.252)$ & \begin{tabular}{|l|}
$0.124(0.134)$ \\
\end{tabular} & \begin{tabular}{|l|}
$0.110(0.023)$ \\
\end{tabular} & $0.093(0.00$ & $13.599(76.040)$ & $0.150(0.108)$ & 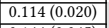 & $0.093(0.00$ & $68.991(234.7$ & \begin{tabular}{|l|l|}
$0.156(0.093)$ \\
\end{tabular} & $0.117(0.032)$ & & t07.0. & $0.170(0.001)$ & & 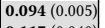 \\
\hline 15 & $4.672(65.606)$ & $0.135(0.058)$ & \begin{tabular}{|l|}
$0.162(0.264)$ \\
\end{tabular} & $0.118(0.003)$ & $16.934(56.442)$ & $0.133(0.071)$ & \begin{tabular}{|l|}
$0.144(0.045)$ \\
\end{tabular} & $0.117(0.028)$ & $65.740(365.449)$ & $0.130(0.071)$ & \begin{tabular}{|l|l|}
$0.152(0.029)$ \\
\end{tabular} & $0.117(0.005)$ & $146.863(561.03$ & $0.168(0.077)$ & \begin{tabular}{|l|}
$0.162(0.030)$ \\
\end{tabular} & $1117(0.0$ \\
\hline 3 & 0.021 & 0.020 & 0.020 & 0.015 & 0.02 & 0.020 & 0.02 & 0.0 & 0.03 & 0.02 & 0.020 & 0.0 & 0.0 & 0.020 & 0.02 & 0.018 \\
\hline & $0.199(0.020)$ & $0.124(0.014)$ & \begin{tabular}{|l|}
$0.122(0.013)$ \\
\end{tabular} & $0.109(0.010)$ & $0.225(0.020)$ & $0.117(0.017)$ & $0.125(0.018)$ & $0.111(0.008)$ & $0.240(0.030)$ & \begin{tabular}{|l|l|}
$0.118(0.015)$ \\
\end{tabular} & \begin{tabular}{|l|l|}
$0.120(0.015)$ \\
\end{tabular} & $0.109(0.009)$ & 0.25 & $0.119(0.017)$ & \begin{tabular}{|l|l}
$0.121(0.020)$ \\
\end{tabular} & $0.113(0.013)$ \\
\hline 0 & $0.619(0.084)$ & \begin{tabular}{|l|}
$0.349(0.025)$ \\
\end{tabular} & \begin{tabular}{|l|}
$0.376(0.039)$ \\
\end{tabular} & $0.323(0.024)$ & $0.662(0.097)$ & $0.350(0.022)$ & \begin{tabular}{|l|}
$0.369(0.037)$ \\
\end{tabular} & 0.328 & $0.700(0.11$ & $0.349(0.031)$ & \begin{tabular}{|l|}
$0.363(0.043)$ \\
\end{tabular} & 0.328 & $0.704(0.12$ & $0.346(0.026)$ & \begin{tabular}{|l}
$0.364(0.041)$ \\
\end{tabular} & 0.33 \\
\hline 15 & $0.862(0.081)$ & $0.490(0.020)$ & \begin{tabular}{|l|}
$0.555(0.071)$ \\
\end{tabular} & $\mathbf{0 . 4 4 0}(0.010)$ & $0.899(0.062)$ & $0.478(0.025)$ & \begin{tabular}{|l|l|}
$0.556(0.079)$ \\
\end{tabular} & $0.433(0.010)$ & $0.911(0.053)$ & $0.468(0.034)$ & \begin{tabular}{|l|l|}
$0.531(0.065)$ \\
\end{tabular} & $0.434(0.010)$ & $0.916(0.078)$ & $0.467(0.032)$ & $0.531(0.065)$ & $\mathbf{0 . 4 3 5}(0.0$ \\
\hline 3 & 0.056 & \begin{tabular}{|l|l|}
1.999 \\
\end{tabular} & 0.02 & 0.021 & $0.156(0.512)$ & 7.99 & 0.0 & 0.0 & $0.238(94.733)$ & 11.99 & 211) & .004 & $1.101(19.266$ & 25.994 & 0.530 & $0.021(0.0$ \\
\hline & $0.450(6.439)$ & \begin{tabular}{|l|}
$0.133(0.024)$ \\
\end{tabular} & \begin{tabular}{|l|}
$0.132(0.036)$ \\
\end{tabular} & $0.121(4$ & $0.639(25.583)$ & $0.126(0.023)$ & \begin{tabular}{|l|}
$0.130(0.029)$ \\
\end{tabular} & 0.1 & 22.298 & \begin{tabular}{|l|l|}
$0.128(0.022)$ \\
\end{tabular} & .026) & 0.12 & 4.629 & $0.022)$ & 030) & $0.119(0.02$ \\
\hline 10 & $0.779(9.148)$ & $0.378(0.025)$ & $24.084(28.582$ & $0.353(0.043)$ & $4.879(53.049)$ & $0.367(0.024)$ & $25.572(32.464)$ & 0.3 & 4.822( & \begin{tabular}{|l|}
$0.372(0.058)$ \\
\end{tabular} & 1.072 & 0. & 29.16 & $0.035)$ & .452 & $0.346(0.02$ \\
\hline 915 & $1.021(27.100)$ & \begin{tabular}{|l|}
$0.820(0.952)$ \\
\end{tabular} & \begin{tabular}{|l}
$2.574(3.491)$ \\
\end{tabular} & $0.473(0.016)$ & $1.267(38.979)$ & $0.615(0.389)$ & $5.145(10.903)$ & $0.468(0.021)$ & 10.864 (119.516) & $0.579(0.101)$ & \begin{tabular}{|l|l}
$4.974(10.807)$ \\
\end{tabular} & $0.471(0.028)$ & $21.122(219.707)$ & $0.561(0.066)$ & $2.516(6.335)$ & $0.466(0.03$ \\
\hline 3 & $0.020(0.003)$ & $0.022(0.003)$ & \begin{tabular}{|l|}
$0.022(0.003)$ \\
\end{tabular} & 0.021 & 0.022( & $0.022(0.003)$ & $\overline{0.021}$ & 0.02 & $\overline{0.02}$ & 004) & 04) & 0.02 & 0.0 & 03) & 04) & 0.02 \\
\hline$\sqrt[3]{5}$ & 0.183 & 014) & 0.1 & & & $\overline{013)}$ & & & & 0.1 & 4) & & & \begin{tabular}{|l|l}
0.1 \\
\end{tabular} & & 0. \\
\hline & 0.564 & $0.392(0.024)$ & 0.386 & 0.374 & $0.835(0.186)$ & $0.395(0.024)$ & 037) & & 1.22 & 027) & 0.38 & & 1.6 & .031) & 0.39 & 0.373 \\
\hline 10 & 98) & $0.495(0.027)$ & 8) & 0.485 & (0) & 33) $>$ & 41) & & 1.353 & & .042) & & 5) & 23) & 0.30 & \\
\hline
\end{tabular}

we employ the generation base $g=8$ as recommended in [6] for real-valued operators and problem variables.

The published implementation of MOEA/D-2TCHMFI [18] employs a specific population size with a specific set of weight vectors for each problem instance. In order to freely scale its population size in the IMS, we replace the provided fixed weight vector sets with the weight vectors generated by the procedure described in Section 3.2. The neighborhood size parameter is set as $10 \%$ of the population size. While the IMS might not be the optimal setting for an MOEA, any MOEA that is integrated with the IMS can be considered as an anytime algorithm, i.e., the longer the MOEA is allowed to run, the better the results will be, and the users can terminate the algorithm whenever they are satisfied with the results.

For all problem instances, each MOEA is run 30 times independently on a multi-core server (4×AMD Opteron Processor 6386 SE, $2.8 \mathrm{GHz}$ ). Note that MO-RV-GOMEAs perform certain tasks (e.g., population clustering, linkage learning), which incur additional computation cost, that MOEA/D-2TCHMFI does not have. For fair comparisons, we thus employ the running time as the computation budget rather than the number of function evaluations. This effectively allows MOEA/D-2TCHMFI to perform more function evaluations than MO-RV-GOMEA. For each problem instance of $m$ objectives and $l$ decision variables, every MOEA is run for $m \times l$ seconds each time. All MOEAs are equipped with an elitist archive, and the target archive size is set empirically as 1000 .

To assess the convergence of each algorithm to the reference Pareto-optimal fronts, we use the Average Front Distance (AFD) [3] that measures the average minimal Euclidean distance over all points in a reference front to an approximation front obtained by an MOEA run. The AFD is also commonly known as the Inverted Generational Distance (IGD) in the literature (e.g., [9, 18]). The median IGD value over the 30 runs of each MOEA is then used as the performance metric. To verify the statistical significance of the obtained results, we use the Mann-Whitney-Wilcoxon statistical hypothesis test for equality of medians with $p<0.05$. In Tables 1,2 , and 3 , the best median value for each problem instance is presented with the gray background, and is further highlighted in bold if the value is statistically significantly better than those obtained by other MOEAs (significance tests are performed with Bonferroni correction).

\section{EXPERIMENTAL RESULTS}

\subsection{DTLZ Problems}

Table 1 shows the medians and the interdecile ranges of the IGD metric of the approximation fronts obtained by MOEAs for the DTLZ1-4 problem instances. In almost all cases, MO-RV-GOMEA outperforms MOEA/D-2TCHMFI, obtaining better approximation fronts. The results in Table 1 confirm that the original design of MO-RV-GOMEA, which yields excellent, and often superior, performance in solving multi-objective problems [6], is also suitable for tackling many-objective problems. This suitability is likely due to the synergy between the multi-objective dominance-based optimization of middle clusters, the single-objective scalar optimization (along each objective) of extreme clusters, and the direct offspring versus parent comparison for accepting improvements in the operation of MO-RV-GOMEA.

The two adaptation options with Tchebycheff scalarization that we propose in this paper assign to each population member a unique improvement region, which could be informative in guiding the search, potentially enhancing the efficiency of MO-RV-GOMEA. Table 1 shows that, for DTLZ problem instances, the performance of the original MO-RV-GOMEA has similar performance (in some cases), or deteriorates (in some cases), or is slightly improved (in other cases) when combined with Option 1 (i.e., using both Tchebycheff-scalarization and Pareto-dominance improvements in the GOM operator). On the other hand, in almost all cases, with Option 2 (i.e., only Tchebycheff-scalarization improvements are checked in the GOM operator), other MOEAs are outperformed, and the differences are found to be statistically significant. Figure 2 shows the median IGD metric convergence of the MOEAs under concern on solving DTLZ1-4 problem instances with $m=3,15$ objectives and $l=320$ decision variables. Except for the cases of DTLZ4, and the case of DTLZ2 with $m=2$, MO-RV-GOMEA adapted with Option 2 achieves the fastest convergence within the allowed computation time. The large obtained IGD values (i.e., IGD $>0.01$ ) indicate that the (reference) Pareto-optimal fronts have not yet been sufficiently reached and more running time would be needed. However, we note that, in this work, we consider problem instances that have many more decision variables, and are thus more difficult, than those considered in recent literature. 


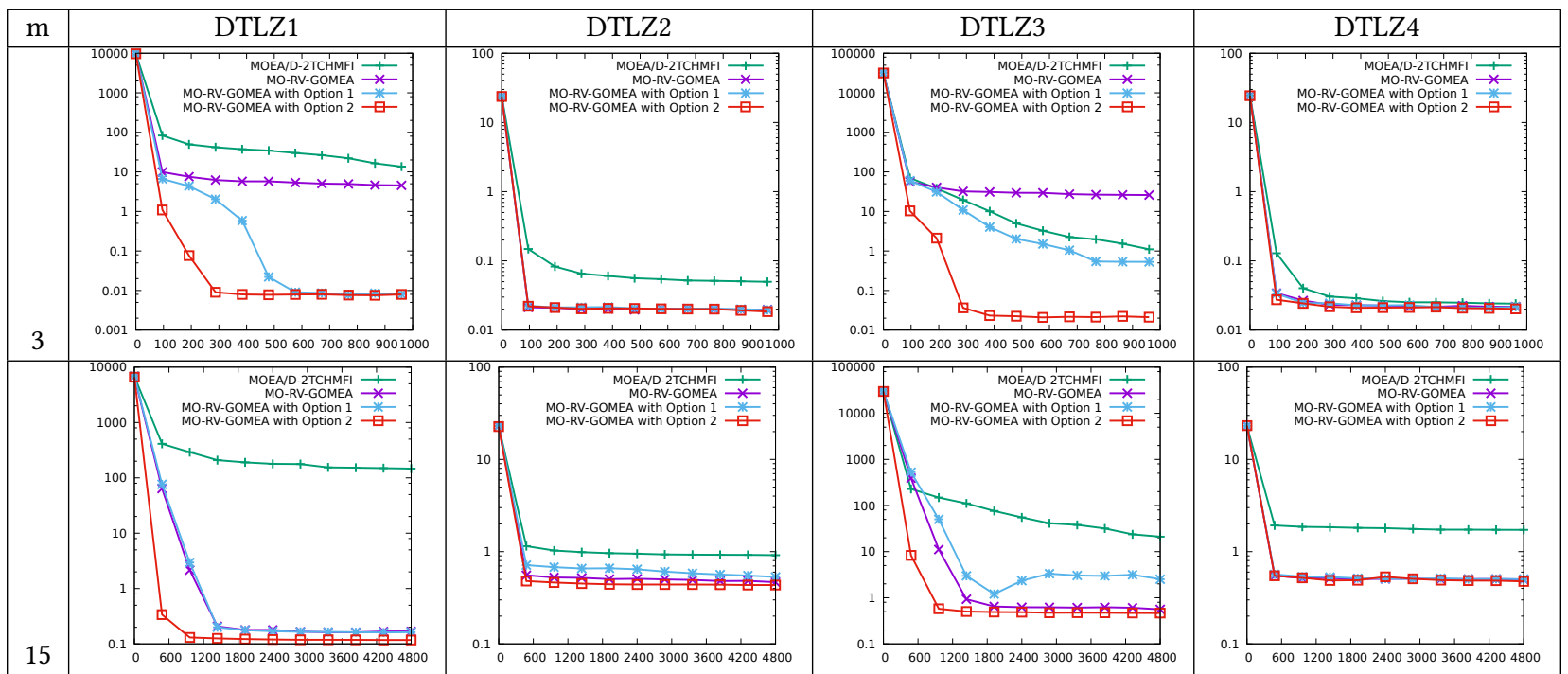

Figure 2: The median IGD convergence on DTLZ1-4 problem instances with 320 variables. Horizonal axis: Time (seconds) in linear scale. Vertical axis: IGD in log scale. Some graphs might appear under another graph due to similar convergence.

Table 2: Medians and interdecile ranges (in brackets) of the IGD metric of the approximation fronts obtained by MOEAs on solving WFG problems. MOEA/D, GOMEA, Option 1, and Option 2 should be read as MOEA/D-2TCHMFI, the original MO-RVGOMEA, MO-RV-GOMEA with adaptation Option 1, and MO-RV-GOMEA with adaptation Option 2, respectively.

\begin{tabular}{|c|c|c|c|c|c|c|c|c|c|c|c|c|c|c|c|c|}
\hline & \multicolumn{4}{|c|}{$l=40$} & \multicolumn{4}{|c|}{$l=80$} & \multicolumn{4}{|c|}{$l=160$} & \multicolumn{4}{|c|}{$l=320$} \\
\hline \multirow[t]{5}{*}{$\mathrm{n}$} & MOEA/D & GOMEA & Option 1 & Option 2 & MOEA/D & GOMEA & Option 1 & Option 2 & MOEA/D & GOMEA & Option 1 & Option 2 & MOEA/D & GOMEA & Option 1 & Option 2 \\
\hline & $1.046(0.056)$ & \begin{tabular}{|l|}
$0.728(0.140)$ \\
\end{tabular} & $0.733(0.091)$ & $0.707(0.216)$ & $1.067(0.056)$ & \begin{tabular}{|l}
$0.784(0.369)$ \\
\end{tabular} & $0.742(0.116)$ & $0.708(0.170)$ & $1.108(0.057)$ & \begin{tabular}{|l}
$0.942(0.829)$ \\
\end{tabular} & $0.748(0.133)$ & \begin{tabular}{|l}
$0.757(0.264)$ \\
\end{tabular} & $1.116(0.075)$ & $\mathbf{0 . 6 4 6}(0.681)$ & $0.708(0.142)$ & $0.771(0.203)$ \\
\hline & $1.434(0.185)$ & $0.740(0.201)$ & \begin{tabular}{|l|}
$0.864(0.246)$ \\
\end{tabular} & \begin{tabular}{|l}
$0.873(0.231)$ \\
\end{tabular} & \begin{tabular}{|l|}
$1.451(0.104)$ \\
\end{tabular} & $0.732(0.358)$ & $0.800(0.196)$ & $0.886(0.293)$ & \begin{tabular}{|l}
$1.475(0.052)$ \\
\end{tabular} & $0.733(0.024)$ & $0.778(0.022)$ & $0.856(0.350)$ & $1.506(0.046)$ & $0.734(0.004)$ & \begin{tabular}{|l|l|}
$0.797(0.132)$ \\
\end{tabular} & $0.411)$ \\
\hline & $1.950(0.047)$ & \begin{tabular}{|l|}
$2.055(1.111)$ \\
\end{tabular} & \begin{tabular}{|l}
$2.352(0.946)$ \\
\end{tabular} & \begin{tabular}{|l}
$2.174(0.779)$ \\
\end{tabular} & $1.947(0.028)$ & $0.996(0.018)$ & \begin{tabular}{|l}
$1.055(0.255)$ \\
\end{tabular} & $1.240(0.548)$ & $1.945(0.051)$ & $0.972(0.303)$ & \begin{tabular}{|l|}
$1.046(0.129)$ \\
\end{tabular} & $1.257(0.371)$ & $1.950(0.021)$ & $0.971(0.130)$ & \begin{tabular}{|l}
$1.085(0.187)$ \\
\end{tabular} & \begin{tabular}{|l}
$1.093(0.204)$ \\
\end{tabular} \\
\hline & $2.850(0.108)$ & 2.083 & \begin{tabular}{|l|}
$2.243(0.905)$ \\
\end{tabular} & $2.497(0.470)$ & $2.864(0.037)$ & $2.147(1.092)$ & $2.241(0.873)$ & \begin{tabular}{|l|}
$2.490(0.743)$ \\
\end{tabular} & $2.890(0.024)$ & $1.586(0.034)$ & $1.702(0.238)$ & $1.770(0.500)$ & $2.891(0.020)$ & $\mathbf{1 . 5 4 4}(0.05$ & \begin{tabular}{|l|}
$1.703(0.110)$ \\
\end{tabular} & $1.832(0.389)$ \\
\hline \multirow{4}{*}{ 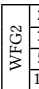 } & $0.009(0.010)$ & $0.237(0.044)$ & $0.052(0.207)$ & \begin{tabular}{|l}
$0.049(0.205)$ \\
\end{tabular} & $\mathbf{0 . 0 4 0}(0.182)$ & \begin{tabular}{|l}
$0.632(0.400)$ \\
\end{tabular} & $0.244(0.403)$ & \begin{tabular}{|l}
$0.110(0.198)$ \\
\end{tabular} & $\mathbf{0 . 2 2 9}(0.019)$ & \begin{tabular}{|l|l}
$0.671(0.035)$ \\
\end{tabular} & $0.635(0.426)$ & $0.243(0.159)$ & $\mathbf{0 . 2 5 3 ( 0 . 0 1 9 )}$ & $0.695(0.030)$ & 0.625( & 0.295 \\
\hline & $0.057(0.014)$ & $0.063(0.026)$ & $0.058(0.022)$ & $0.057(0.016)$ & $0.096(0.071)$ & $0.090(0.036)$ & $0.086(0.045)$ & $0.081(0.051)$ & $0.134(0.173)$ & $0.154(0.168)$ & $0.137(0.117)$ & $0.339(0.321)$ & \begin{tabular}{|l|}
$0.312(0.037)$ \\
\end{tabular} & $0.223(0.166)$ & $\mathbf{0 . 1 7 6}(0.222)$ & \begin{tabular}{|l|l}
$0.416(0.546)$ \\
\end{tabular} \\
\hline & $0.226(0.037)$ & $0.171(0.040)$ & \begin{tabular}{|l|}
$0.172(0.043)$ \\
\end{tabular} & \begin{tabular}{|l|l|}
$0.177(0.032)$ \\
\end{tabular} & $0.236(0.049)$ & \begin{tabular}{|l}
$0.186(0.054)$ \\
\end{tabular} & $0.187(0.054)$ & $0.177(0.027)$ & $\mid 0.276(0.101)$ & $0.270(0.052)$ & $0.252(0.055)$ & $0.225(0.063)$ & $0.640(0.389)$ & $0.342(0.078)$ & \begin{tabular}{|l|}
$0.319(0.080)$ \\
\end{tabular} & $0.275(0.088)$ \\
\hline & $0.793(0.484)$ & $1.299(0.635)$ & $1.173(0.656)$ & $1.774(0.447)$ & $0.972(0.886)$ & $1.377(0.810)$ & $1.191(0.812)$ & \begin{tabular}{|l}
$1.783(0.469)$ \\
\end{tabular} & $2.418(0.686)$ & $1.837(0.292)$ & \begin{tabular}{|l}
$1.894(0.330)$ \\
\end{tabular} & \begin{tabular}{|l|}
$2.099(0.336)$ \\
\end{tabular} & $2.641(0.688)$ & $\begin{array}{l}2.031(0.362) \\
\end{array}$ & $1.976(0.292)$ & \begin{tabular}{|l|}
$2.235(0.157)$ \\
\end{tabular} \\
\hline & 0.0 & $0071(0054)$ & 0.0 & 0.0 & 0.6 & $0.116(0.059)$ & \begin{tabular}{|l|l}
$0.078(0.074)$ \\
\end{tabular} & & 0. & 0.1 & 0. & & & & & 0. \\
\hline & $0.100(0.047)$ & $0.054(0.028)$ & $0.043(0.030)$ & $\mathbf{0 . 0 3 7}(0.017)$ & $0.145(0.061)$ & $0.093(0.047)$ & $0.060(0.034)$ & $0.043(0.020)$ & $0.193(0.048)$ & $0.111(0.045)$ & $0.054(0.044)$ & $0.043(0.026)$ & $0.216(0.055)$ & $0.128(0.048)$ & $0.056(0.035)$ & $0.051(0.025)$ \\
\hline & $0.261(0.161)$ & $0.188(0.052)$ & $\mathbf{0 . 1 4 9}(0.042)$ & \begin{tabular}{|l|}
$0.184(0.062)$ \\
\end{tabular} & $0.414(0.210)$ & \begin{tabular}{|l|l|}
$0.190(0.066)$ \\
\end{tabular} & $0.129(0.071$ & \begin{tabular}{|l|l|l}
$0.164(0.068)$ \\
\end{tabular} & 0.485 & \begin{tabular}{|l}
$0.234(0.077)$ \\
\end{tabular} & 0.113 & \begin{tabular}{|l}
$0.126(0.094)$ \\
\end{tabular} & $0.470(0.174)$ & $0.242(0.064)$ & $\mathbf{0 . 1 0 5}(0.046)$ & 0.145 \\
\hline & $0.810(0.165)$ & \begin{tabular}{|l|}
$0.342(0.154)$ \\
\end{tabular} & $0.331(0.237)$ & \begin{tabular}{|l|}
$0.360(0.164)$ \\
\end{tabular} & $0.819(0.141)$ & $0.460(0.219)$ & $0.395(0.218)$ & \begin{tabular}{|l}
$0.401(0.189)$ \\
\end{tabular} & \begin{tabular}{|l|l|}
$0.807(0.100)$ \\
\end{tabular} & $\begin{array}{l}0.443(0.219) \\
\end{array}$ & \begin{tabular}{|l|}
$0.439(0.246)$ \\
\end{tabular} & $0.348(0.143)$ & \begin{tabular}{|l|l}
$0.786(0.133)$ \\
\end{tabular} & \begin{tabular}{|l|}
$0.561(0.238)$ \\
\end{tabular} & $0.566(0.207)$ & $0.393(0.161)$ \\
\hline \multirow{4}{*}{$\begin{array}{l}\text { 总 } \\
\text { 索 }\end{array}$} & $0.003(0.005)$ & $0.002(0.001)$ & $0.002(0.001)$ & 0.002(0.001) & $0.003(0.003)$ & $0.003(0.001)$ & $0.003(0.002)$ & $0.002(0.001)$ & $0.011(0.015)$ & $0.002(0.003)$ & $0.001(0.000)$ & $0.002(0.001)$ & $0.030(0.024)$ & $0.001(0.003)$ & $0.001(0.000)$ & $0.001(0.000)$ \\
\hline & $0.076(0.010)$ & $0.090(0.008)$ & $0.090(0.012)$ & $0.078(0.011)$ & $0.115(0.051)$ & $0.089(0.014)$ & $0.092(0.006)$ & $0.078(0.008)$ & \begin{tabular}{|l|}
$0.158(0.143)$ \\
\end{tabular} & $0.095(0.016)$ & $0.085(0.021)$ & $\mathbf{0 . 0 7 6}(0.009)$ & $0.293(0.208)$ & $0.112(0.026)$ & $0.095(0.027)$ & $0.077(0.010)$ \\
\hline & $0.622(0.018)$ & \begin{tabular}{|l|}
$0.753(0.073)$ \\
\end{tabular} & $0.763(0.078)$ & $0.701(0.065)$ & $0.730(0.064)$ & $0.802(0.082)$ & $0.823(0.073)$ & $\mathbf{0 . 6 1 4}(0.065)$ & \begin{tabular}{|l|l|}
$1.019(0.427)$ \\
\end{tabular} & $0.852(0.095)$ & $0.862(0.072)$ & $\mathbf{0 . 6 3 0}(0.062)$ & $1.641(0.235)$ & \begin{tabular}{|l|l|}
$0.967(0.079)$ \\
\end{tabular} & $0.928(0.078)$ & $0.662(0.072)$ \\
\hline & $3.205(0.158)$ & \begin{tabular}{|l|}
$3.852(0.300)$ \\
\end{tabular} & $3.798(0.273)$ & \begin{tabular}{|l}
$3.441(0.283)$ \\
\end{tabular} & $3.130(0.119)$ & \begin{tabular}{|l|}
$3.891(0.382)$ \\
\end{tabular} & $3.789(0.558)$ & \begin{tabular}{|l|}
$3.475(0.286)$ \\
\end{tabular} & \begin{tabular}{|l|l}
$3.828(0.179)$ \\
\end{tabular} & \begin{tabular}{|l}
$4.025(0.617)$ \\
\end{tabular} & $4.038(0.649)$ & $\mathbf{3 . 1 9 4}(0.214)$ & $4.150(0.163)$ & \begin{tabular}{|l|l}
$4.249(0.599)$ \\
\end{tabular} & \begin{tabular}{|l|l}
$4.231(0.411)$ \\
\end{tabular} & $3.340(0.194)$ \\
\hline
\end{tabular}

\subsection{WFG Problems}

Table 2 shows the medians and the interdecile ranges of the IGD metric of the approximation fronts obtained by MOEAs on solving WFG1-4 problem instances. For WFG1, the original MO-RVGOMEA obtains the best IGD values in almost all cases. For WFG2-4, MOEA/D-2TCHMFI obtains better IGD values when solving instances that have $l=40$ decision variables while the two adapted MO-RV-GOMEA variants obtain better results when solving larger problem instances that have $l=80,160,320$ decision variables. Figure 3 shows the median IGD convergence of the MOEAs under concern in solving WFG problem instances with $m=2,10$ objectives and $l=320$ decision variables. MO-RV-GOMEA with adaptation Option 2 has the best IGD convergence for the WFG3-4 instances with the most objectives (i.e., $m=10$ ) while the original MO-RV-GOMEA exhibits the best performance for WFG1. Similar to the case of DTLZ problems, better results can be obtained if more running time is allowed. From the results in Tables 1 and 2, we can conclude that, in most cases, adaptation Option 2 is better than Option 1 for improving the performance of MO-RV-GOMEA. We hypothesize that a key factor in the outcomes is that removing checks for acceptance into the archive, which is expensive if the archive is full (1000 solutions), allows MO-RV-GOMEA with adaptation Option 2 to perform many more evaluations (empirically we found up to a factor of 5). Note that the Tchebycheff scalarizations play a non-trivial role here, as simply removing the archive check in GOM in the original MO-RV-GOMEA deteriorates its performance.

Figure 4 shows three approximation fronts with median IGD values obtained by MOEA/D-2TCHMFI, the original MO-RV-GOMEA, and MO-RV-GOMEA with adaptation Option 2, respectively, for the WFG4 problem instance with $m=3$ objectives and $l=320$ decision variables. While the two MO-RV-GOMEAs both reach the reference front, there is still a considerable gap between the approximation front obtained by MOEA/D-2TCHMFI and the reference front. The 


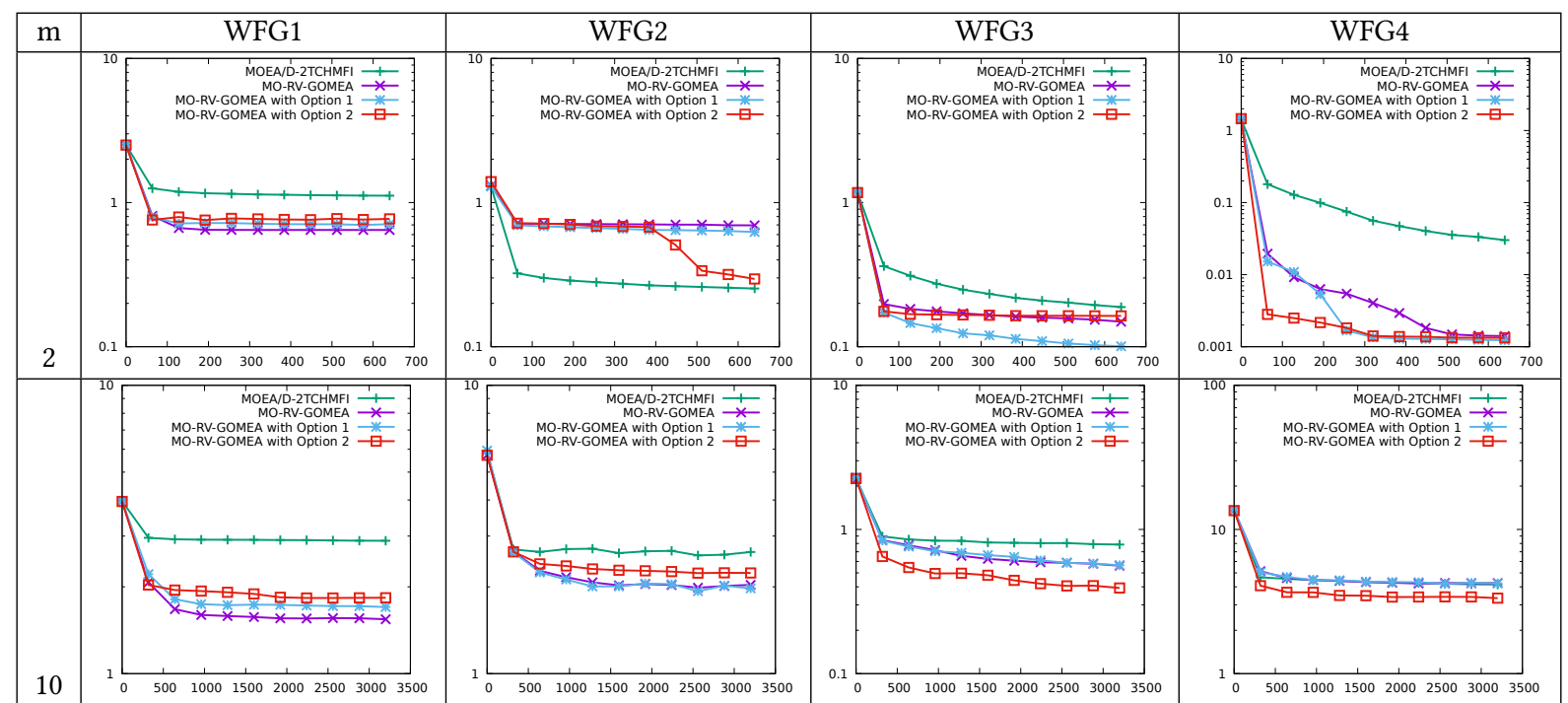

Figure 3: The median IGD convergence on WFG1-4 problem instances with 320 variables. Horizonal axis: Time (seconds) in linear scale. Vertical axis: IGD in log scale. Some graphs might appear under another graph due to similar convergence.

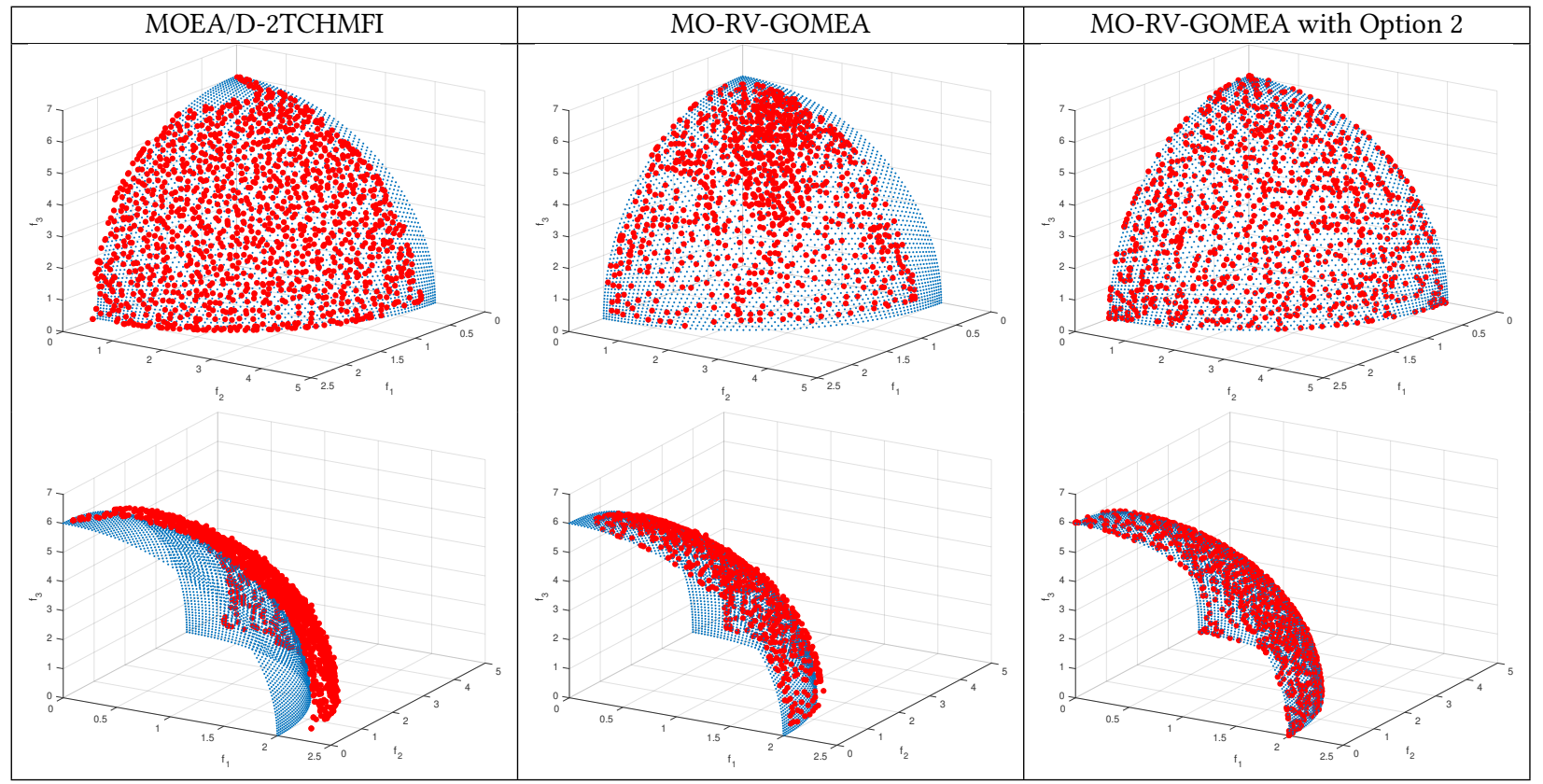

Figure 4: Approximation fronts (in red) with the median IGD values on the WFG4 problem instance with $m=3$ and $l=320$ (the reference Pareto-optimal fronts are in blue).

front obtained by MO-RV-GOMEA with adaptation Option 2 contains the extreme solutions and is more well-spread than the one of the original MO-RV-GOMEA.

The results presented above have been obtained by MO-RVGOMEAs with the univariate model, i.e., all decision variables are considered as independent from each other. In MOEA/D-2TCHMFI, the polynomial mutation and simulated binary crossover (SBX) are employed to generate offspring solutions. While these two operators are also univariate, they still have certain differences compared to the variation operator of MO-RV-GOMEAs. More detailed analysis would require experimenting with SBX in MORV-GOMEAs as well to recognize separately the contribution of scalarizations from the contribution of variation operators to the performance of MO-RV-GOMEAs compared to MOEA/D-2TCHMFI, which is outside the scope of this paper.

WFG2-3 are non-separable problems [11], i.e., their decision variables exhibit certain dependencies that need to be properly handled during solution variations so that the problem instances 
Table 3: Results on solving WFG2-3 problems with MO-RV-GOMEAs using the linkage tree model.

\begin{tabular}{|c|c|c|c|c|c|c|c|c|c|c|c|c|c|c|c|c|c|}
\hline \multirow{2}{*}{\multicolumn{2}{|c|}{\begin{tabular}{|l|l|} 
& \\
& $\mathrm{m}$ \\
\end{tabular}}} & \multicolumn{4}{|c|}{$l=40$} & \multicolumn{4}{|c|}{$l=80$} & \multicolumn{4}{|c|}{$l=160$} & \multicolumn{4}{|c|}{$l=320$} \\
\hline & & MOEA/D & GOMEA & Option 1 & ption 2 & MOEA/D & GOMEA & Option 1 & Option 2 & MOEA/D & GOMEA & Option 1 & Option 2 & MOEA/D & GOMEA & Option 1 & Option 2 \\
\hline & 2 & $0.009(0.010)$ & $\overline{0.001}(0.001)$ & $0.002(0.001)$ & $0.001(0.000)$ & $0.040(0.182)$ & \begin{tabular}{|l|}
$0.194(0.193)$ \\
\end{tabular} & \begin{tabular}{|l|}
$\mathbf{0 . 0 0 1}(0.002)$ \\
\end{tabular} & $.002(0.193)$ & & $0.606(0.413)$ & $0.194(0.413)$ & $0.194(0.413)$ & $0.253(0.019)$ & \begin{tabular}{|l|}
$0.606(0.000)$ \\
\end{tabular} & $0.606(0.413)$ & $0.400(0.413)$ \\
\hline & 3 & \begin{tabular}{|l|l|}
$0.057(0.014)$ \\
\end{tabular} & $0.046(0.015)$ & $0.048(0.013)$ & $0.053(0.018)$ & $0.096(0.071)$ & $0.053(0.023)$ & & 3) & .173) & $0.061(0.027)$ & 0.06 & 0.05 & 0.312 & & 190) & $0.062(0.238)$ \\
\hline & \begin{tabular}{|l|}
5 \\
\end{tabular} & $0.226(4$ & $0.165(0.044)$ & 0.180 & 0.198 & & \begin{tabular}{|l|}
$0.184(0.035)$ \\
\end{tabular} & $0.182(0.052)$ & $182(0.051)$ & 0.276 & \begin{tabular}{|l|l|l}
$0.217(0.068)$ \\
\end{tabular} & & $0.179(0.032)$ & 0.640 & $0.288(0.085)$ & \begin{tabular}{|l|}
$0.270(0.049)$ \\
\end{tabular} & .078) \\
\hline & & $0.793(0.484)$ & $1.207(0.763)$ & $1.331(0.528)$ & \begin{tabular}{|l}
$1.321(0.928)$ \\
\end{tabular} & $0.972(0.886)$ & \begin{tabular}{|l|}
$1.160(0.911)$ \\
\end{tabular} & \begin{tabular}{|l|}
$1.309(1.097)$ \\
\end{tabular} & \begin{tabular}{|l|}
$1.329(0.796)$ \\
\end{tabular} & $2.418(0.686)$ & $1.685(0.682)$ & $1.447(0.926)$ & \begin{tabular}{|l|}
$2.037(0.264)$ \\
\end{tabular} & 2.64 & $2.139(0.434)$ & $1.966(0.490)$ & $2.057(0.308)$ \\
\hline & 0. & $0.003(0.002)$ & 0.0 & 0. & 0.068 & 0. & 006 & & & & & & & $.000)$ & & \\
\hline & & $0.100(0.047)$ & $0.031(0.009)$ & 0.034 & 0.026 & 0.14 & 0.030 & & & & & & & & & & \\
\hline & & 0.261 & 0.199 & 0.177 & 0.124 & 0.41 & $0.171(0.068)$ & 0.16 & 0.1 & 0.48 & 0.14 & & 43) & 0.4 & 95) & & 36) \\
\hline & & $0.810(0.165)$ & $0.401(0.195)$ & $0.329(0.283)$ & $\mathbf{0 . 1 8 7}(0.085)$ & $0.819(0.141)$ & $0.342(0.265)$ & \begin{tabular}{|l|}
$0.384(0.507)$ \\
\end{tabular} & $0.216(0.136)$ & $\mid 0.807(0.100)$ & $0.359(0.272)$ & $0.235(0.184)$ & $0.228(0.064)$ & $0.786(0.133)$ & $0.326(0.269)$ & $0.317(0.401)$ & $0.190(0.080)$ \\
\hline
\end{tabular}

can be efficiently solved. Table 3 shows the medians and the inderdecile ranges of the IGD metric of the approximation fronts obtained by the three MO-RV-GOMEA variants that employ the linkage tree model, which is learned over the selection of solutions in each generation to capture the linkage structures, and the MOEA/D-2TCHMFI. We do not implement the linkage tree model for MOEA/D-2TCHMFI because non-trivial modifications would be required for MOEA/D-2TCHMFI (which, by default, creates a whole offspring solution each time) to make use of the linkage tree. In most cases, MO-RV-GOMEAs with the linkage tree model obtain better IGD values compared to the ones with the univariate model (i.e., compare Table 2 and Table 3). For WFG2 problem instances with $l=80,160,320$ variables, the two adapted MO-RVGOMEAs achieve better approximation fronts than the original MO-RV-GOMEA and MOEA/D-2TCHMFI. For WFG3 problem instances, MO-RV-GOMEA with Option 2 almost always outperforms other MOEAs. A more detailed analysis on the effect of linkage learning (for such large problem instances) requires more computation time and thus is left for future work.

\section{CONCLUSIONS}

We investigated the performance of the MO-RV-GOMEA on a wide range of multi-objective and many-objective benchmark problems. The experimental results indicate that MO-RV-GOMEA is quite wellsuited also for solving many-objective problems without requiring any modification, unlike many other MOEAs that are designed on the basis of multi-objective optimization. For some benchmark problems, the performance of MO-RV-GOMEA can be further improved when the improvement checks during solution variation are guided by carefully constructed Tchebycheff scalarizations. Performance comparisons with a state-of-the-art decomposition-based MOEA (i.e., MOEA/D-2TCHMFI) show that both the original and adapted MO-RV-GOMEAs are the preferred algorithms of choice for tackling (real-world) problems that require many conflicting objectives to be optimized at the same time.

\section{ACKNOWLEDGMENTS}

This work is part of the research programme IPPSI-TA with project number 628.006.003, which is financed by the Netherlands Organisation for Scientific Research (NWO) and Elekta.

\section{REFERENCES}

[1] M. Asafuddoula, Tapabrata Ray, and Ruhul Sarker. 2015. A decomposition-based evolutionary algorithm for many objective optimization. IEEE Transactions on Evolutionary Computation 19, 3 (2015), 445-460.

[2] Leonardo C. T. Bezerra, Manuel López-Ibáñez, and Thomas Stützle. 2016. A performance assessment of tuned multi- and many-objective evolutionary algorithms. (2016). http://iridia.ulb.ac.be/supp/IridiaSupp2015-007/.
[3] Peter A.N. Bosman and Dirk Thierens. 2002. Multi-objective optimization with diversity preserving mixture-based iterated density estimation evolutionary algorithms. International fournal of Approximate Reasoning 31, 3 (2002), 259-289.

[4] Peter A. N. Bosman. 2010. The anticipated mean shift and cluster registration in mixture-based EDAs for multi-objective optimization. In Proceedings of the Genetic and Evolutionary Computation Conference - GECCO 2010. ACM Press, New York, New York, USA, 351-358.

[5] Peter A. N. Bosman and Marcus Gallagher. 2016. The importance of implementation details and parameter settings in black-box optimization: a case study on Gaussian estimation-of-distribution algorithms and circles-in-a-square packing problems. Soft Computing (2016), 1-15.

[6] Anton Bouter, Ngoc Hoang Luong, Cees Witteveen, Tanja Alderliesten, and Peter A. N. Bosman. 2017. The multi-objective real-valued gene-pool optimal mixing evolutionary algorithm. In Proceedings of the Genetic and Evolutionary Computation Conference - GECCO 2017. 537-544.

[7] Indraneel Das and John E. Dennis. 1997. A closer look at drawbacks of minimizing weighted sums of objectives for Pareto set generation in multicriteria optimization problems. Structural Optimization 14, 1 (1997), 63-69.

[8] Indraneel Das and John E. Dennis. 1998. Normal-Boundary Intersection: a new method for generating the Pareto surface in nonlinear multicriteria optimization problems. SIAM Journal on Optimization 8, 3 (1998), 631-657.

[9] Kalyanmoy Deb and Himanshu Jain. 2014. An evolutionary many-objective optimization algorithm using reference-point-based nondominated sorting approach, Part I: solving problems with box constraints. IEEE Transactions on Evolutionary Computation 18, 4 (2014), 577-601.

[10] Kalyanmoy Deb, Lothar Thiele, Marco Laumanns, and Eckart Zitzler. 2005. Scalable Test Problems for Evolutionary Multiobjective Optimization. In Evolutionary Multiobjective Optimization. Springer-Verlag, London, 105-145.

[11] Simon Huband, Phil Hingston, Luigi Barone, and Lyndon While. 2006. A review of multiobjective test problems and a scalable test problem toolkit. IEEE Transactions on Evolutionary Computation 10, 5 (2006), 477-506.

[12] Hisao Ishibuchi, Ken Doi, and Yusuke Nojima. 2016. Characteristics of manyobjective test problems and penalty parameter specification in MOEA/D. In 2016 IEEE Congress on Evolutionary Computation (CEC). IEEE, 1115-1122.

[13] Joshua Knowles and David Corne. 2003. Properties of an adaptive archiving algorithm for storing nondominated vectors. IEEE Transactions on Evolutionary Computation 7, 2 (2003), 100-116.

[14] Ke Li, Kalyanmoy Deb, Qingfu Zhang, and Sam Kwong. 2015. An evolutionary many-objective optimization algorithm based on dominance and decomposition. IEEE Transactions on Evolutionary Computation 19, 5 (2015), 694-716.

[15] Fernando G. Lobo and David E. Goldberg. 2004. The parameter-less genetic algorithm in practice. Information Sciences 167, 1 (2004), 217-232.

[16] Ngoc Hoang Luong, Tanja Alderliesten, Arjan Bel, Yury Niatsetski, and Peter A.N. Bosman. 2017. Application and benchmarking of multi-objective evolutionary algorithms on high-dose-rate brachytherapy planning for prostate cancer treatment. Swarm and Evolutionary Computation (2017). https://doi.org/10.1016/j. swevo.2017.12.003

[17] Ngoc Hoang Luong and Peter A. N. Bosman. 2012. Elitist archiving for multiobjective evolutionary algorithms: to adapt or not to adapt. In Parallel Problem Solving from Nature - PPSN XII, Vol. 7492. Springer Berlin Heidelberg, 72-81.

[18] Xiaoliang Ma, Qingfu Zhang, Junshan Yang, and Zexuan Zhu. 2017. On Tchebycheff decomposition approaches for multi-objective evolutionary optimization. IEEE Transactions on Evolutionary Computation (2017), 1-20.

[19] Qingfu Zhang and Hui Li. 2007. MOEA/D: A multiobjective evolutionary algorithm based on decomposition. IEEE Transactions on Evolutionary Computation 11, 6 (2007), 712-731.

[20] Dirk Thierens and Peter A. N. Bosman. 2011. Optimal mixing evolutionary algorithms. In Proceedings of the Genetic and Evolutionary Computation Conference - GECCO 2011. ACM Press, New York, New York, USA, 617-624

[21] Ye Tian, Ran Cheng, Xingyi Zhang, and Yaochu Jin. 2017. PlatEMO: A MATLAB platform for evolutionary multi-objective optimization [Educational Forum]. IEEE Computational Intelligence Magazine 12, 4 (2017), 73-87.

[22] Anupam Trivedi, Dipti Srinivasan, Krishnendu Sanyal, and Abhiroop Ghosh. 2017. A survey of multiobjective evolutionary algorithms based on decomposition. IEEE Transactions on Evolutionary Computation 21, 3 (2017), 440-462. 